\title{
Urinary excretion of apo(a) fragments in NIDDM patients
}

\author{
M. Clodii ${ }^{1}$, R. Oberbauer ${ }^{2}$, W. Waldhäusl ${ }^{1}$, G. Maurer ${ }^{3}$, G. M. Kostner ${ }^{4}$, K. Kostner ${ }^{3}$ \\ ${ }^{1}$ Third Department of Medicine, Division of Endocrinology, University of Vienna, Austria \\ ${ }^{2}$ Third Department of Medicine, Division of Nephrology, University Hospital of Vienna, Austria \\ ${ }^{3}$ Second Department of Medicine, Division of Cardiology, University Hospital of Vienna, Austria \\ ${ }^{4}$ Department of Medical Biochemistry, University of Graz, Austria
}

\begin{abstract}
Summary $\mathrm{Lp}(\mathrm{a})$, one of the most atherogenic lipoproteins, is believed to contribute significantly to vascular diseases in non-insulin-dependent diabetic (NIDDM) patients. Contradictive data have been published on these patients concerning plasma concentrations of $\mathrm{Lp}(\mathrm{a})$ and their relation to renal function. Since apo(a) fragments appear in urine, we measured urinary apo(a) in 134 NIDDM patients and 100 matched controls and related urinary apo(a) concentrations to plasma Lp(a) levels and kidney function. Plasma $L p(a)$ values were found to be significantly higher in NIDDM patients. NIDDM patients also secreted significantly more apo(a) into their urine as compared to control subjects. There was no correlation between creatinine clearance or albumin
\end{abstract}

excretion and urinary apo(a) concentrations. Patients with macroalbuminuria exhibited a twofold higher apparent fractional excretion of apo(a) in comparison to patients with normal renal function. Urinary apo(a) values in both patients and control subjects were highly correlated to plasma $\mathrm{Lp}(\mathrm{a})$, yet no correlation was found with $\mathrm{HbA}_{1 \mathrm{c}}$ or serum lipoproteins. It is concluded that urinary apo(a) excretion is correlated to plasma $L p(a)$ levels but not to creatinine clearance in patients suffering from NIDDM. [Diabetologia (1997) 40: 1455-1460]

Keywords NIDDM, Lp(a), urinary apo(a) excretion, microalbuminuria, macroalbuminuria.
Due to the strong correlation between high plasma $\mathrm{Lp}$ (a) levels and coronary artery disease, $\mathrm{Lp}(\mathrm{a})$ has been established as an independent risk factor for atherosclerotic disease, stroke and peripheral atherosclerosis [1-5]. In addition, $\mathrm{Lp}(\mathrm{a})$ has appeared as a predictor of myocardial infarction in several case-control as well as prospective studies [2-6]. Protein and cDNA sequencing of apo(a) revealed a high degree of homology to plasminogen [7] and by inhibiting plasminogen activation in a templatedependent manner, $\operatorname{Lp}(\mathrm{a})$ was found to inhibit

Received: 26 May 1997 and in revised form: 21 July 1997

Corresponding author: Dr. K. Kostner, AKH Wien, Department of Cardiology, Währingergürtel 18-20, A-1090 Vienna, Austria

Abbreviations: NIDDM, Non-insulin-dependent diabetes mellitus; AER, albumin excretion rate; FE, fractional excretion rate. fibrinolysis [8]. $\mathrm{Lp}$ (a) also shows a high affinity to proteoglycans, which may be the reason that it is found in high amounts in atherosclerotic plaques [9]. Thus Lp(a) has become one of the most widely studied lipoproteins in atherosclerosis research. Despite this flourish of activity, little is known concerning the physiological function and catabolism of $\operatorname{Lp}(\mathrm{a})$.

There is an excess of coronary artery disease in patients with non-insulin-dependent diabetes mellitus (NIDDM), which has been attributed partially to the atherogenic lipoprotein pattern in these patients [10-14]. In many studies, subjects with NIDDM are reported to have elevated plasma levels of $\mathrm{Lp}(\mathrm{a})$ [15-17]. Those findings, however, are contradictive as there are also numerous studies where Lp(a) plasma levels of NIDDM patients were not different from those in control subjects [18-20].

Recently it was published that apo(a) immunoreactivity is found in urine [21-25]. However, it is not 
intact $L p(a)$ that appears in urine, but rather fragments of the apo(a) antigen. Independent of the apo(a) isoform, more than 10 distinct apo (a) bands have been consistently found in urine in our own investigations with molecular masses between 50-160 $\mathrm{kDa}$ and with a rather characteristic electrophoretic banding pattern. These apo (a) fragments were glycosylated and not complexed to apoB. We also reported a highly significant correlation between urinary apo(a) concentrations normalized to creatinine levels, and plasma $\mathrm{Lp}(\mathrm{a})$ levels $(R=0.68, p<0.001)$ in 193 healthy volunteers [22].

In this study we investigated the excretion of urinary apo(a) fragments in 134 NIDDM patients with different grades of diabetic nephropathy and compared them to 100 non-diabetic healthy control subjects.

\section{Materials and methods}

Patients and control subjects. We recruited 134 subjects with NIDDM from the Outpatient Diabetes Unit of the University Hospital Vienna. The inclusion criteria were: 1) known diabetes duration of at least 12 months; 2) age at onset of diabetes 35 years or over; 3 ) no use of insulin in the first year after diagnosis; 4) no history of ketoacidosis and 5) current treatment with either diet, oral hypoglycaemic agents or insulin. Urinary tract infections were excluded by urine microscopy and culture. A total of 61 patients received insulin substitution and 51 received oral hypoglycemic agents. The remaining patients were treated only by dietary restrictions. In order to assess the influence of diabetic nephropathy on plasma Lp(a) and urinary apo(a) levels, the patients were assigned to three groups according to albumin excretion rate (AER), as determined by 24-h urine sampling (group I: AER $<20 \mu \mathrm{g} / \mathrm{min}$; group II: AER $>20<200 \mu \mathrm{g} / \mathrm{min}$; group III: AER $>200 \mu \mathrm{g} / \mathrm{min}$ ). The control group consisted of 100 non-diabetic, healthy subjects, matched for age, sex and socioeconomic status during a university health survey programme. All investigations were performed with the consent of each patient and according to the Declaration of Helsinki.

Demographic data of NIDDM patients and control subjects are provided in Table 1.

Blood and urine samples. Following an overnight fasting period, blood samples were taken from the antecubital vein, allowed to clot for $30 \mathrm{~min}$ at room temperature, centrifuged and the serum was frozen at $-20^{\circ} \mathrm{C}$. In previous experiments we ascertained that freezing once did not affect the assays performed. Urine, collected in the morning, was also frozen at $-20^{\circ} \mathrm{C}$ and assayed in one run together with the serum samples. For investigating the apo(a) fragments, urine was concentrated by pressure dialysis by a factor of approximately 100 and immediately processed further.

Immunoquantitation of $\operatorname{Lp}(a)$ and apo(a). Analyses were carried out by a sandwich Dissociation Enhanced Lantanoid Fluorescence Immuno Assay (DELFIA) essentially as recommended by the manufacturer, LKB-Pharmacia (Vienna, Austria) and described in detail previously [22]. For the determination of total apo(a), europium (Eu) labelled polyclonal (POAB) anti-apo(a) antibody from rabbit was used ( = a-a
Table 1. Demographic data of NIDDM patients and control subjects

\begin{tabular}{llll}
\hline & Patients & $\begin{array}{l}\text { Control } \\
\text { subjects }\end{array}$ & Significance \\
\hline Number & 134 & 100 & - \\
Male/Female & $74 / 60$ & $58 / 42$ & N.S. \\
Age (years) & $63 \pm 11$ & $58 \pm 16$ & N.S. \\
BMI $\left(\mathrm{kg} / \mathrm{m}^{2}\right)$ & $28.4 \pm 4.6$ & $24.1 \pm 5.5$ & $p<0.005$ \\
Coronary artery disease & 37 & - & \\
Peripheral vascular & & & \\
disease & 11 & - & - \\
Smokers & 21 & 27 & N.S. \\
Diabetes duration (year) & $10.8 \pm 8.6$ & - & \\
Alcohol consumption & $<20 \mathrm{~g} /$ day & $<20 \mathrm{~g} /$ day & N.S. \\
HbA $_{1 \mathrm{c}}(\%)$ & $8.0 \pm 1.3$ & $4.3 \pm 1.2$ & $p<0.005$ \\
\hline
\end{tabular}

Data are mean \pm SD

DELFIA). Apo(a):apoB complexes were measured using Eulabelled anti-apoB antibody from rabbit as detection antibody (a-B DELFIA) as described previously [22]. For measuring total apoB, an apoB:apoB DELFIA was designed accordingly [22]. Plasma samples were diluted 3000-fold and urine samples were diluted between 10-50-fold. The standardization of the DELFIA assay was performed by using highly purified primary standards consisting of recombinant apo(a), Lp(a) or LDL or freshly frozen secondary standards as described [22]. The accuracy of the DELFIA assays was ascertained by spiking fresh plasma or urine samples with purified $L p(a)$ or recombinant apo(a) and measuring the yield of the added analyte [22]. Yields were in the range of 96-106\%. The assay was linear between 1 and $100 \mathrm{ng}$ of apo(a) per well; the within-run coefficient of variation was less than $3 \%$.

Apo(a) concentration in urine was normalized to creatinine where it is stated in the text.

Polyacrylamide gel electrophoresis (PAGE) and Western blotting was carried out as described previously [22].

Total cholesterol, HDL cholesterol, triglycerides and albumin were determined with commercially available kits from Boehringer Mannheim (Mannheim, Germany). LDL cholesterol was calculated using the Friedewald equation. In patients with plasma triglyceride levels above $5 \mathrm{mmol} / \mathrm{l}$, direct measurement of LDL-cholesterol after precipitation of HDL and VLDL, was performed with commercially available kits from Boehringer Mannheim.

Creatinine was measured by the Jaffee Method using commercial assay kits from Boehringer Mannheim.

All chemicals were reagent grade from E.Merck (Darmstadt, Germany) if not stated otherwise.

Statistical analysis. The analysis of our data was performed by the Statistical Package for Social Sciences (SPSS/MAc +). For serum lipids, mean values \pm SEM were calculated and analysed by a one-way variance test (ANOVA). A Student's $t$ test was applied to assess significant differences of continuous variables among groups. Comparison of serum $\mathrm{Lp}(\mathrm{a})$ and urine apo(a) values among groups were performed by the Wilcoxon test or by ANOVA after logarithmic transformation of values. Correlations of serum apo(a) and urine apo(a) values were performed by the Spearmann Rank Correlation Test. 


\section{Results}

Demographic data of patients and control subjects are shown in Table 1 . There was no significant difference in age, smoking habits and alcohol consumption between patients and control subjects. Of the patients $28 \%$ suffered from coronary artery disease and $8.5 \%$ from peripheral vascular disease. No such diseases were evident in the control group.

Although it was not the aim of this study to reveal differences in plasma lipoproteins between NIDDM patients and control subjects, some of the lipid and lipoprotein values are displayed in Table 2 . Total cholesterol, triglycerides and LDL-cholesterol were higher in patients, and HDL-cholesterol was higher in control subjects. All differences were statistically significant.

As described previously, apo(a) immunereactivity is found in the urine of healthy individuals in the form of apo(a) fragments. Even in individuals with virtually no detectable plasma $\mathrm{Lp}(\mathrm{a})$, urinary apo(a) fragments can be detected [22]. This immunereactivity consists of apo(a) fragments, i.e. mainly apo(a) kringle-IV type 1 and type- 2 domains, ranging in molecular weight from 50 to $160 \mathrm{kDa}$. In patients with NIDDM, immunoblotting determined that apo(a) fragments were present with features identical to those described for the normal population (Fig.1).

In order to reveal possible quantitative differences between diabetic patients and control subjects, we measured $\mathrm{Lp}(\mathrm{a})$ in plasma and apo(a) in morning urine normalized to $100 \mathrm{mg} / \mathrm{dl}$ of creatinine in 134 NIDDM patients and compared them with that of a control group (Table 3). The 25th and 50th percentile values of plasma $\mathrm{Lp}(\mathrm{a})$ concentrations were comparable in patients $(5.3$ and $12.7 \mathrm{mg} / \mathrm{dl})$ and control subjects $(6.0$ and $12.0 \mathrm{mg} / \mathrm{dl}$, respectively). The 75 th percentile was much higher in patients compared to control subjects ( 34.9 vs $21.3 \mu \mathrm{g} / \mathrm{dl}$ ). Overall plasma $\mathrm{Lp}(\mathrm{a})$ levels were significantly higher in patients than in control subjects as calculated by the Wilcoxon signed rank test (Table 3). When we compared plasma $L p(a)$ levels in patients with and without albuminuria, we did not observe a significant difference. Patients with macroalbuminuria did not have elevated plasma $\mathrm{Lp}(\mathrm{a})$ levels when compared to control subjects.

Urinary apo(a) excretion was then measured. On comparison, NIDDM patients exhibited strikingly higher urinary apo(a) concentrations than control subjects. The median values and the 75 th percentiles of urinary apo(a) were approximately twice as high in patients than in control subjects $(37.8$ and $67.7 \mu \mathrm{g} /$ dl vs 18.0 and $37.7 \mu \mathrm{g} / \mathrm{dl}$ respectively). More importantly, however, was the finding that the 25 th percentile apo(a) concentrations of patients were 6 times higher than those of control subjects (22.9 vs $3.8 \mu \mathrm{g} /$ dl respectively). The difference in urinary apo(a)

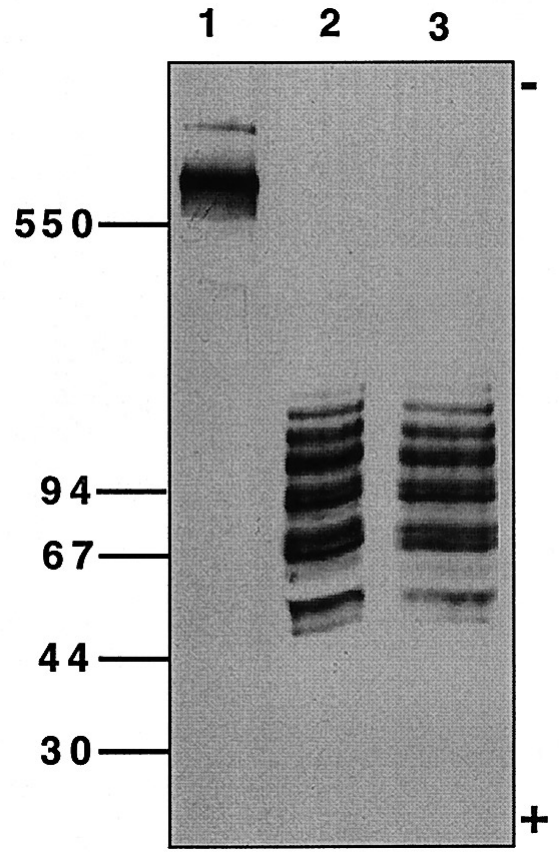

Fig. 1. Western blot of 1: plasma, 2: urine from an NIDDM patient and 3: urine from a control individual. Samples were electrophoresed in 3-25\% SDS-PAGE followed by transblotting to nitro cellulose. The blot was developed with polyclonal anti-apo(a) from rabbit as described in Methods. The numbers on the left mark the position of molecular weight markers in $\mathrm{kDa}$

Table 2. Lipid and lipoprotein concentrations of NIDDM patients and matched control subjects

\begin{tabular}{lllll}
\hline & Triglycerides & Cholesterol & LDL-C & HDL-C \\
\hline Patients $n=134$ & $2.8 \pm 4.0$ & $5.7 \pm 1.6$ & $3.45 \pm 1.0$ & $1.0 \pm 0.4$ \\
Control subjects & & & & \\
$n=100$ & $1.2 \pm 0.6$ & $4.9 \pm 1.2$ & $3.2 \pm 0.8$ & $1.2 \pm 0.4$ \\
$p$-value & 0.001 & 0.0004 & 0.03 & 0.03 \\
\hline
\end{tabular}

Values are mean $\pm \mathrm{SD}$. All values given in $\mathrm{mmol} / \mathrm{l} . p$ value by ANOVA (except for triglycerides, which were tested by Wilcoxon signed rank test)

excretion between patients and control subjects was statistically highly significant (Table 3 ). To answer the question as to how the hyperlipidaemic state among some of the NIDDM subjects might affect the plasma $\operatorname{Lp}(\mathrm{a})$ levels and the urinary apo(a) excretion, we performed a sub-group analysis by creating lipid matched sub-groups and comparing normolipidaemic healthy subjects with normolipidaemic NIDDM subjects. Both sub-groups had total cholesterol values less than $5.5 \mathrm{mmol} / \mathrm{l}$ and triglycerides less than $2.5 \mathrm{mmol} / \mathrm{l}$. Plasma Lp(a) levels were significantly higher in patients as compared to control subjects. (patients: $n=62$, median: $12.8 \mathrm{mg} / \mathrm{dl}, 25$ th percentile: $4.9 \mathrm{mg} / \mathrm{dl}$, 75 th percentile: $31.7 \mathrm{mg} / \mathrm{dl}$; control subjects: $n=86$, median: $11.8 \mathrm{mg} / \mathrm{dl}, 25$ th percentile: $6.1 \mathrm{mg} / \mathrm{dl}, 75$ th percentile: $21.2 \mathrm{mg} / \mathrm{dl}, p<0.001$ ). 
Table 3. Plasma Lp(a) and urinary apo(a) values of NIDDM patients and control subjects

\begin{tabular}{|c|c|c|c|c|c|c|c|}
\hline \multirow[t]{2}{*}{ Group } & \multirow[t]{2}{*}{$n$} & \multicolumn{3}{|c|}{ Plasma Lp(a) (mg/dl) } & \multicolumn{3}{|c|}{ Urine apo(a) $(\mu \mathrm{g} / \mathrm{dl})^{\mathrm{a}}$} \\
\hline & & $25 \%$ & $50 \%$ & $75 \%$ & $25 \%$ & $50 \%$ & $75 \%$ \\
\hline Patients & 134 & 5.3 & 12.7 & 34.9 & 22.9 & 37.8 & 67.7 \\
\hline $\begin{array}{l}\text { Control } \\
\text { subjects }\end{array}$ & 100 & 6.0 & 12.0 & 21.3 & 3.8 & 18.0 & 37.7 \\
\hline$z$ test $^{\mathrm{b}}$ & & & -12.7 & & & -12.0 & \\
\hline$p$ value $^{\mathrm{b}}$ & & & 0.001 & & & 0.000 & \\
\hline
\end{tabular}

${ }^{a}$ Normalized to $100 \mathrm{mg} / \mathrm{dl}$ creatinine

b Tested by Wilcoxon signed rank test: NIDDM patients vs control subjects

Urinary apo(a) excretion also revealed higher values in NIDDM patients when compared to control subjects (patients: median: $42.3 \mu \mathrm{g} / \mathrm{dl}, 25$ th percentile: $25.3 \mu \mathrm{g} / \mathrm{dl}$, 75 th percentile: $63.1 \mu \mathrm{g} / \mathrm{dl}$; control subjects: median: $17.8 \mu \mathrm{g} / \mathrm{dl}$, 25th percentile: $4.0 \mu \mathrm{g} /$ $\mathrm{dl}$, 75th percentile: $37.3 \mathrm{mg} / \mathrm{dl}, p<0.0001)$. From these data we concluded that the observed differences in plasma $\mathrm{Lp}(\mathrm{a})$ and urinary apo(a) levels were not biased by differences in the plasma lipid content.

In order to determine whether diabetic nephropathy was responsible for the higher plasma $L p(a)$ and urinary apo(a) levels in NIDDM patients, we correlated these parameters with kidney function. For this, creatinine clearance in the NIDDM patient group was correlated with urinary apo(a) concentrations. These two parameters were not significantly correlated. This was also true for the urinary albumin excretion and urinary apo(a) levels. The urinary excretion of albumin in $\mu \mathrm{g} / \mathrm{min}$ was not significantly related to the measured urinary apo(a) concentrations $(R=0.122)$.

Because of the great variation of urinary apo(a) levels, even in the normal population [22], it was more important to see whether the apparent fractional excretion of apo(a) might be related to kidney function. In order to do this, we calculated the apparent fractional excretion rate (FE) of urinary apo(a) according to the equation:

$\{$ urinary apo(a) $\times$ plasma creatinine/plasma apo(a) $\times$ urinary creatinine $\}$ and divided the collective of patients into three groups: Patients with a urinary albumin excretion of $<20 \mu \mathrm{g} / \mathrm{min} \quad(n=52)$; patients with microalbuminuria: urinary albumin excretion of $20-200 \mu \mathrm{g} / \mathrm{min}(n=33)$ and patients with macroalbuminuria: urinary albumin excretion $>200 \mu \mathrm{g} / \mathrm{dl}$ $(n=45)$. Patients with a urinary albumin excretion of $<20 \mu \mathrm{g} / \mathrm{min}$ had an FE of $0.02 \%$, while patients with microalbuminuria had an $\mathrm{FE}$ of $0.016 \%$ and patients with macroalbuminuria had an FE of $0.035 \%$. The FE of apo(a) was significantly higher in patients with macroalbuminuria $(p<0.02)$ as compared to the other two groups.
We previously published that urinary apo(a) values are strongly correlated with plasma $L p(a)$ in the normal population [22]. Here we were interested to see whether this might also be true for NIDDM patients. We found a highly significant correlation not only in control subjects but also in the NIDDM patient group as measured by the Spearman rank correlation test $(p<0.001)$. In contrast, we did not find a significant correlation of $\mathrm{HbA}_{1}$, cholesterol, triglycerides and urinary protein excretion with either plasma $\mathrm{Lp}(\mathrm{a})$ or with urinary apo(a).

\section{Discussion}

Numerous papers have been published concerning the impact of diabetes mellitus on plasma Lp(a) concentrations [15-20, 31, 32]. The major questions asked in these studies were: i) are plasma Lp(a) levels altered in IDDM and NIDDM patients in comparison to control subjects? ii) Does metabolic control have an influence on fluctuations of plasma $\mathrm{Lp}(\mathrm{a})$ levels? iii) Are the reported increased plasma $L p(a)$ levels in diabetic patients related to impaired kidney function? iv) Are increased plasma levels in diabetic patients a cause for the high atherogenicity in this patient group. On reviewing these studies, conflicting results are evident; taking them together it seems that there is no difference from diabetes per se and that only diabetics with decreased kidney function show increased plasma Lp(a) levels. Undoubtedly, further research in this area is needed to prove these assumptions.

Recently, we and others reported that apo(a) fragments with molecular masses between 50 and 215 $\mathrm{kDa}$ are secreted into urine [22-25]. Apo(a) fragments were found to be free of apo B and urinary apo(a) levels were significantly correlated to urinary creatinine levels in a given individual [22-25]. Although the exact mechanism of apo(a) excretion by the kidney remains to be determined, it is concluded from earlier studies that apo(a) excretion is constant over months [22] and that these fragments may be actively secreted by the tubulus [23, 24, 32].

It was the major goal of this study to investigate urinary apo(a) fragments in NIDDM individuals and to correlate the concentration of apo(a) with that of plasma $L p(a)$. Since numerous studies indicate that the kidney plays a key role in Lp(a) catabolism [2630] we also asked whether kidney function has some impact on urinary apo(a) excretion in NIDDM patients.

In our study, plasma Lp(a) levels were significantly higher in the 134 NIDDM patients as compared to matched control subjects, and this was independent from plasma cholesterol and triglyceride values. It was of interest to note however, that median and 25 th percentile values of patients and control subjects 
were comparable and only in the 75th percentile $\mathrm{Lp}$ (a) was approximately $50 \%$ higher in patients than in control subjects. Even when we excluded patients with macrovascular disease, we found significantly higher values in our NIDDM patients. In comparison to other studies [33] we did not find a difference in plasma $\mathrm{Lp}(\mathrm{a})$ levels between patients with and without albuminuria. Taken as a whole group a significant elevation was calculated in NIDDM patients by the Wilcoxon signed rank test. As we reported previously that urinary apo(a) excretion is highly correlated with plasma $\mathrm{Lp}(\mathrm{a})$ levels in normal subjects, we anticipated that urinary apo(a) concentrations might also be increased in diabetic patients. This was in fact the case, however a much more striking increase in apo(a) was found in urine of diabetic patients than in control subjects which covered all percentiles. Particularly in the 25th percentile, urinary apo(a) in NIDDM patients was six times higher than in control subjects. In this respect, it is noteworthy that we and others $[22,25,34]$ found that urinary apo(a) levels are not linearly correlated with plasma $\mathrm{Lp}(\mathrm{a})$ in individuals with low plasma $\mathrm{Lp}(\mathrm{a})$ levels but rather that individuals with low plasma Lp(a) excrete a proportionally higher amount of apo(a) fragments into urine. Although the metabolic mechanisms leading to the excretion of apo(a) by the kidney remain to be determined, from our study it appears that the "basal" apo(a) excretion in NIDDM is much higher than in control subjects.

Next we asked whether kidney function has any impact on urinary apo(a) excretion. Neither creatinine clearance nor urinary albumin excretion was significantly correlated with urinary apo(a) levels in the whole patient group. This was not surprising, as there is a great variation of urinary apo(a) levels even in the normal population which highly depends on their plasma Lp(a) levels [22]. In fact this relationship was also true for NIDDM patients with similar results compared to control subjects. It will be of interest to study the relationship of urinary apo(a) to creatinine clearance and urinary albumin excretion longitudinally in single individuals. Such studies are currently being conducted at our department.

A more direct way to answer the question as to whether impaired kidney function influences apo(a) excretion is the measurement of the apparent fractional excretion of apo(a) into urine. As we reported previously [22] urinary apo(a) fragments account for only approximately $1 \%$ of the daily $\mathrm{Lp}(\mathrm{a})$ catabolism which is only a minute fraction of the circulating apo(a) pool. Fractional urinary excretion among individuals is highly variable, and therefore the difference in FE between NIDDM patients with normal kidney function and with microalbuminuria $(0.02 \%$ and $0.016 \%$ respectively) may not be considered as significant. In the group of patients with macroalbuminuria however, FE was $0.035 \%$, which was higher than any value observed in individuals with normal kidney function. Thus once diabetic nephropathy occurs, apo(a) secreted into urine increases independently of the plasma Lp(a) level. The fact that urinary protein loss in diabetic patients is a valuable indicator of glomerular function and that we did not find a correlation between urinary apo(a) excretion and protein excretion points towards the possibility that apo(a) is selectively excreted via the tubulus. The reason why we found a considerable increase in the FE of apo(a) in patients with macroalbuminuria who excreted more than $200 \mathrm{mg}$ of protein per minute could reflect a tubular damage in these patients.

It was not the aim of this study to answer the question as to what extent NIDDM may influence plasma $\mathrm{Lp}(\mathrm{a})$, but in our collective we found no correlation between $\mathrm{HbA}_{1 \mathrm{c}}$ and plasma Lp(a) or urinary apo(a) which was probably due to large population fluctuations of these parameters. In order to study such correlations single individuals would have to be studied over prolonged periods.

In the present study we also did not study apo(a) isoform distribution in patients and control subjects, since two large previous studies reported that NIDDM patients have larger apo(a) size because of considerable glycation of apo(a), yet the genotype distribution appeared to be identical in patients and control subjects $[17,35]$.

In conclusion, we show here that NIDDM patients have, in addition to higher plasma levels of $\mathrm{Lp}(\mathrm{a})$ as compared to control subjects, significantly higher urinary apo(a) values, and this is independent of plasma lipids. Further studies will indicate whether or not the measurement of urinary apo(a) in NIDDM may be of clinical relevance in this patient group.

Acknowledgements. The expert technical assistance of Anton Ibovnic is kindly appreciated. Parts of this work were supported by grants of the Austrian Research Foundation, grant number P-11691 and the Austrian National Bank, grant \# 5956 to GMK.

\section{References}

1. Berg K, Dahlen G, Frick MH (1974) Lp(a) lipoprotein and pre- $\beta 1$-lipoprotein in patients with coronary heart disease. Clin Genet 6: 230-235

2. Kostner GM, Avogaro P, Cazzolato G, Marth E, Bittolobon G (1981) Lipoprotein Lp(a) and the risk for myocardial infarction. Atherosclerosis 38: 51-61

3. Armstrong VW, Cremer P, Eberle E et al. (1986) The association between serum $\mathrm{Lp}(\mathrm{a})$ concentrations and angiographically assessed coronary atherosclerosis. Atherosclerosis 62: 249-257

4. Murai A, Miyahara T, Fujimoto N, Matsuda M, Kameyana M (1986) Lp(a) lipoprotein as a risk factor for coronary heart disease and cerebral infarction. Atherosclerosis 59: 199-204

5. Cushing GL, Gaubatz JW, Nava ML et al. (1989) Quantitation and localization of apolipoprotein(a) and B in 
coronary artery bypass vein grafts resected at re-operation. Arteriosclerosis 9: 593-603

6. Cremer P, Nagel D, Labrot B et al. (1994) Lp(a) as predictor of myocardial infarction in comparison to fibrinogen, LDL-C and other risk factors: results from prospective Göttingen Risk Incidence and Prevalence Study (GRIPS). Eur J Clin Invest 24: 444-453

7. McLean JW, Tomlinson JE, Kuang WJ et al. (1987) cDNA sequence of human apolipoprotein (a) is homologous to plasminogen. Nature 300: 132-137

8. Edelberg JM, Pizzo SV (1991) Lp(a) inhibits plasminogen activation in a template dependent manner. Blood Coagul Fibrinolysis 2: 759-764

9. Bihari-Varga M, Gruber E, Rotheneder M, Zechner R, Kostner GM (1988) The Interaction of lipoprotein (a) and LDL with glycosamino glycans and proteoglycans from human aorta. Arterioscler Thromb 8: 851-857

10. Kannel WB, McGee DC (1979) Diabetes and cardiovascular risk factors: the Framingham study. Circulation 59: 8-13

11. Assmann G, Schulte H (1988) The prospective cardiovascular Munster (PROCAM) Study: prevalence of hyperlipidaemia in persons with hypertension and diabetes mellitus and the relationship to coronary heart disease. Am Heart J 116: $1713-1724$

12. Haffner SM, Mosse SE, Klein BEK, Klein R (1992) Lack of association between lipoprotein(a) concentrations and coronary heart disease mortality in diabetes: the Wisconsin epidemiologic study of diabetic retinopathy. Metabolism 41: 194-197

13. O'Brien T, Nguyen CTT, Harrison JM, Bailey KR, Dyck PJ, Kottke BA (1994) Lipids and Lp(a) lipoprotein levels and coronary artery disease in subjects with NIDDM. Mayo Clin Proc 69: 430-435

14. Hiraga T, Kobayashi T, Okubo M et al. (1995) Prospective study of lipoprotein(a) as a risk factor for atherosclerotic cardiovascular diseases in patients with diabetes. Diabetes Care 18: 241-244

15. Kikuchi T, Onuma T, Shimura M et al. (1994) Different change in lipoprotein (a) levels from lipid levels of other lipoproteins with improved glycemic control in patients with NIDDM. Diabetes Care 17: 1059-1061

16. Heesen BJ, Wolfenbuttel BH, Leurs PB et al. (1993) Lipoprotein (a) levels in relation to diabetic complications in patients with non insulin dependent diabetes. Eur J Clin Invest 23: 580-584

17. Csaszar A, Dieplinger H, Sandholzer C et al. (1993) Plasma lipoprotein(a) concentrations and phenotypes in diabetes mellitus. Diabetoligia 36: 47-51

18. Rainwater DL, MacCluer JW, Stern MP, VandeBerg JL, Haffner SM (1994) Effects of NIDDM on lipoprotein(a) concentration and apolipoprotein(a) size. Diabetes 43: 942-946

19. Haffner S, Morales PA, Stern M, Gruber MK (1992) Lp(a) concentrations in NIDDM. Diabetes 41: 1266-1272
20. Kuusi T, Yki Jarvinen H, Kauppinen et al. (1995) Effect of insulin treatment on serum lipoprotein (a) in non insulin dependant diabetes. Eur J Clin Invest 25: 194-200

21. Oida K, Takai H, Maeda H et al. (1992) Apolipoprotein (a) is present in urine and its excretion is decreased in patients with renal failure. Clin Chem 38/11: 2244-2248

22. Kostner K, Maurer G, Huber K et al. (1996) Urinary excretion of apo(a) fragments: role in apo(a) catabolism. Atheroscl Thromb 16/8: 905-911

23. Mooser V, Miguel CS, Moeen A, Landschulz K, Marcovina S, Hobbs HH (1996) Apolipoprotein(a) kringle 4-containing fragments in human urine. J Clin Invest 97: 858-864

24. Kostner K, Jansen M, Maurer G, Derfler K (1997) LDLapheresis significantly reduces urinary apo(a) excretion. E J Clin Invest 27: 93-95

25. Kostner K, Huber K, Stefenelli T, Maurer G (1997) Urinary apo(a) discriminates between $\mathrm{CAD}$ and controls. Atherosclerosis 129: 103-110

26. Parra HJ, Mezdour H, Cachera C, Dracon M, Tacquet A, Fruchart JC (1987) Lp(a) lipoprotein in patients with chronic renal failure treated by hemodialysis. Clin Chem 33: 721-722

27. Karadi I, Romics L, Palos G et al. (1989) Lp(a) concentration in serum of patients with heavy proteinuria of different origin. Clin Chem 35: 2121-2123

28. Faucher C, Doucet C, Baumelou A, Chapman J, Jacobs C, Thillet J (1993) Elevated Lp(a) levels in primary nephrotic syndrome. Am J Kidney Dis 22: 808-813

29. Thomas ME, Freestone A, Varghese Z, Persaud JW, Moorhead JF (1992) Lp(a) in patients with proteinuria. Nephrol Dial Transplant 7: 597-601

30. Kronenberg F, Utermann G, Dieplinger H (1996) Lipoprotein (a) in renal disease. American Journal of Kidney Diseases 27: $1-25$

31. Sidhu M, Crook D, Godsland IF, Walton C Wynn V, Oliver MF (1992) Inverse relationship between serum Lp(a) levels and first phase insulin secretion. Diabetes 41: 1341-1345

32. Levitsky LL, Scanu AM, Gould SH (1991) Lipoprotein(a) levels in black and white children and adolescents with IDDM. Diabetes Care 14: 283-287

33. Jenkins AJ, Steele JS, Janus AD, Santamaria JD, Best JD (1992) Plasma apolipoprotein (a) is increased in type 2 (non-insulin-dependent) diabetic patients with microalbuminuria. Diabetologia 35: 1055-1059

34. Mooser V, Marcovina S, White A, Hobbs H (1996) Kringlecontaining fragments of apolipoprotein (a) circulate in human plasma and are excreted into urine. J Clin Invest 10: 2414-1424

35. Rainwater DL, MacCluer JW, Stern MP, VandeBerg JL, Haffner SM (1994) Effects of NIDDM on lipoprotein (a) concentration and apolipoprotein size. Diabetes. 43: 942946 